\title{
Compuestos de Poliéster Reforzados con Fibra de Plátano/Banano (Musa paradisiaca) Modificada Químicamente. Comparación con Fibra de Vidrio y Fique (Furcraea andina)
}

\author{
Lady J. Rodríguez(1), William A. Sarache(1) y Carlos E. Orrego ${ }^{(2)^{*}}$ \\ (1) Universidad Nacional de Colombia, Sede Manizales, Departamento de Ingeniería Industrial, Campus la \\ Nubia Km 4 Vía al Magdalena, AA 127 Manizales, Colombia (e-mail: ljrodriguezs@unal.edu.co; \\ wasarachec@unal.edu.co) \\ (2) Universidad Nacional de Colombia, Sede Manizales, Instituto de Biotecnología y Agroindustria, \\ Departamento de Física y Química, Campus la Nubia Km 4 Vía al Magdalena, AA 127, Manizales, \\ Colombia. (e-mail: corregoa@unal.edu.co)
}

* Autor a quien debe ser dirigida la correspondencia

Recibido Ene. 30, 2014; Aceptado Abr. 22, 2014; Versión final recibida May. 7, 2014

\section{Resumen}

Se fabricaron piezas de materiales compuestos mediante la técnica de moldeo manual con fibras del seudo tallo de plátano (FSP), fique y vidrio en una matriz de poliéster. Las FSP fueron tejidas y analizadas sin tratamiento y tratadas químicamente con anhídrido acético, epiclorhidrina y su mezcla. El objetivo fue caracterizar y comparar los compuestos obtenidos mediante ensayos de tensión, impacto, capacidad de absorción de agua y micrografías. El valor promedio de la tensión a la ruptura de los materiales compuestos con FSP tratadas aumentó respecto a las FSP sin tratamiento y al fique. Además presentaron el menor incremento de peso en las pruebas de absorción de agua. Este comportamiento se atribuyó a una mayor hidrofobicidad de las FSP tratadas. Esto a su vez produjo mejor adhesión con la matriz, como se corroboró en las micrografías que mostraron menor deslizamiento en las superficies de corte.

Palabras clave: fibra de banano/plátano, anhídrido acético, epiclorhidrina, compuesto de poliéster

\section{Polyester Composites Reinforced with Banana/plantain (Musa paradisiaca) Fiber Chemically Modified. Comparison with Fiberglass and Fique (Furcraea andina)}

\begin{abstract}
Samples of composites were prepared using the hand lay-up technique with banana seudo-stem fibers (BSF), fique and glass, in a polyester matrix. The BSF was woven and analyzed untreated and chemically treated with acetic anhydride, epichlorohydrin, and their mixtures. The aim of this work was to characterize and compare the composites obtained using tensile tests, impact test, water absorption capacity and micrographs. The average value of tensile strength for the composite with treated BSF increased respect to untreated BSF and fique. Also, according to the water absorption tests, they showed the smallest weight increase. This finding was attributed to the higher hydrophobicity of the treated BSF. This in turn improved the adhesion with the matrix, as it was corroborated by the micrographs that showed lower displacement on the tensile fracture surfaces.
\end{abstract}

Keywords: banana-plantain fiber, acetic anhydride, epichlorohydrin, polyester composite 


\section{INTRODUCCIÓN}

El actual interés por la protección del medio ambiente y el desarrollo sostenible han fomentado las investigaciones en temas como el reciclaje, la disminución de las emisiones de gases y el uso de materias primas de origen natural, renovables y biodegradables (Flieger, et al., 2003, Mohanty, et al., 2005). Las fibras naturales tales como el algodón, el lino, la seda y el sisal entre otras, conformadas principalmente por biopolímeros, han sido utilizadas desde hace muchos años, especialmente en la industria textil. Su producción es una actividad que se puede incluir dentro del enfoque de economía verde, por ser materiales de origen natural, renovables y, al final de su ciclo de vida, completamente biodegradables (Flieger, et al., 2003, Johansson, et al., 2012, Mohanty, et al., 2005). Además, su producción, procesamiento y comercialización benefician a millones de agricultores y procesadores, principalmente de países en vía de desarrollo (FAO, 2009). Colombia es uno de los mayores productores en el mundo de banano y plátano (Musa paradisiaca), productos en los que actualmente más del $85 \%$ de su biomasa es desperdiciada en zona de cultivo (Rodríguez, et al., 2011). Buena parte de estos materiales son potencialmente aprovechables para la extracción de las fibras del seudotallo de plátano (FSP), de características similares a las de fique (Furcraea andina) y el yute.

Gracias a mejoras tecnológicas que proporcionan propiedades específicas, los materiales compuestos que incorporan fibras naturales como relleno, pueden llegar a soportar altas cargas estructurales, lo cual los convierte en materiales de gran utilidad en muchas áreas. Se destacan las aplicaciones en la industrias de la construcción y automotriz, sistemas de liberación controlada, ingeniería de tejidos biológicos y empaques para alimentos. Para su aplicación óptima en materiales compuestos, las fibras naturales habitualmente se modifican química o físicamente. Entre las modificaciones físicas realizadas a las variedades se destacan los tratamientos con plasma DBD (Oliveira, et al., 2012) y con vapor (Gutiérrez, et al., 2005), gracias a las cuales se han logrado mejoras significativas en la adhesión de las fibras con las matrices poliméricas. Dos productos químicos que pueden usarse para modificar fibras son la epiclorhidrina y el anhídrido acético. El primero de ellos ha sido utilizado como entrecruzador de los grupos $\mathrm{OH}$ de biopolímeros (Kuniak, et al., 1972, Zeng, et al., 1996), y no ha sido usado en fibras naturales, mientras que el anhídrido acético permite reemplazar estos grupos por acetilos. En ambos casos las fibras se tornan más hidrofóbicas e insolubles en agua (Freier, et al., 2005, D.-Y. Kim, et al., 2002).

En el presente trabajo se probaron los efectos de tratamientos superficiales realizados a las FSP (Musa paradisiaca) con una combinación de epiclorhidrina y anhídrido acético para reducir su afinidad por el agua, característica que hace que la fibra sea fácilmente atacada por agentes químicos y biológicos, con la consecuente reducción de sus propiedades mecánicas y su vida útil. Se fabricaron bajo el mismo procedimiento de moldeo manual de piezas de MC en matriz de poliéster con FSP tejidas, crudas y tratadas, fique y vidrio. Estos materiales fueron evaluados por diferentes técnicas de caracterización y tales atributos fueron comparados. Los resultados permitieron comprobar que las FSP modificadas redujeron significativamente su afinidad por el agua, lo que les proporcionó una mejor adhesión con la matriz polimérica, evidenciado por la disminución en la absorción de agua y el aumento de la rigidez de sus MC.

\section{MATERIALES Y MÉTODOS}

\section{Preparación de las Fibras}

Las FSP crudas fueron obtenidas del seudotallo de plátano (musa paradisiaca), recolectado de los cultivos del Municipio de Caicedonia (Valle, Colombia). El seudotallo se cortó después de la cosecha y, mediante un proceso de desfibrado manual o mecánico, se extrajeron las fibras que luego fueron cepilladas para remover los residuos, secadas naturalmente hasta una humedad de $13 \pm 2 \%$. Antes de usarlas en la fabricación de MC, las FSP fueron hiladas y tejidas manualmente por artesanos de la misma región y se almacenaron a una temperatura de $20^{\circ} \mathrm{C}$ y $62 \pm 2 \%$ de humedad relativa. Las fibras de fique (tejido) y de vidrio (tela) fueron compradas en el mercado local.

\section{Reactivos}

La epiclorhidrina (EP) y el anhídrido acético (AA), fueron comprados en Panreac (España). La resina de poliéster insaturada ortoftálica (semi-preparada) y el catalizador (Peróxido de Benzoilo), fueron comprados en Industrias Macar S.A. (Palmira, Colombia).

\section{Tratamientos químicos}

Los químicos (AA, EP y AA_EP) fueron disueltos en acetona (proporción de químico: acetona, 1:10 y proporción en peso AA en EP 1:1). Las fibras crudas (proporción en peso de fibras crudas: químico, 1:20) fueron inmersas en una solución de químico/acetona durante 24 horas, a $20^{\circ} \mathrm{C}$. Después, las fibras fueron 
lavadas varias veces en suficiente acetona y agua destilada para asegurar la remoción de todos los residuos de los reactivos. Finalmente, las fibras tratadas fueron secadas en un horno a $105^{\circ} \mathrm{C}$ por $24 \mathrm{~h}$.

\section{Preparación de los compuestos}

Los compuestos fueron elaborados con las FSP tejidas $\left(0.246 \pm 0.001 \mathrm{~g} / \mathrm{cm}^{2}\right)$ impregnadas con resina poliéster y peróxido de benzoilo como catalizador (proporción en peso poliéster y catalizador 100:2; porcentaje de fibra en el compuesto, 15\%). Para preparar las piezas de materiales compuestos (MC), fue usada la técnica de moldeo manual. Unas placas de acero (dimensiones $230 \mathrm{~mm} \times 200 \mathrm{~mm}$ ) fueron cubiertas con desmoldante y el tejido de la FSP fue cortado a medida y puesto sobre la placa. Las fibras fueron completamente impregnadas con la resina preparada. Después otra placa fue puesta encima, se cerró el módulo y se sometió a un proceso de prensado durante 1 hora en la que ocurrió el proceso de solidificación (presión estimada de $4.5 \mathrm{MPa}$ ). Posteriormente, el compuesto se curó por 24 horas a temperatura ambiente $\left(20^{\circ} \mathrm{C}\right)$.

\section{Prueba de tensión}

Las pruebas de tensión fueron realizadas en una máquina universal de tensión AG-X marca Shimazu (capacidad de carga $50 \mathrm{kN}$ ), humedad relativa $65 \pm 5 \%$ y $23 \pm 1^{\circ} \mathrm{C}$. Cinco muestras de cada tipo de $\mathrm{MC}$ fueron preparadas de acuerdo con la norma internacional ISO 527-4. El equipo operó con una velocidad de 20 $\mathrm{mm} / \mathrm{min}$ y un rango de carga de $1500 \mathrm{~N}$. Se analizaron el esfuerzo máximo de tracción, la elongación en el momento del rompimiento o deformación de corte y el módulo de Young.

\section{Prueba de impacto}

La prueba de impacto fue evaluada en una máquina Ceast Ce-man 022 (Turín-Italia) con una potencia máxima de $11 \mathrm{~J}$ y sensibilidad de 0.02 . Cinco muestras de cada tipo fueron preparadas de acuerdo con la norma internacional ASTM-D256-10. Las probetas fueron cortadas a $64 \times 13 \times 5 \mathrm{~mm}$ con muesca. La probeta de ensayo fue apoyada por una viga en voladizo vertical con la muesca de frente al péndulo y fueron rotas por una única oscilación. De esta manera se obtuvo la resistencia al impacto o tenacidad de los especímenes de MC de poliéster forzado con FSP, fique y vidrio. Las pruebas se hicieron a temperatura ambiente.

\section{Absorción de agua}

A los especímenes fabricados con las distintas fibras se les midió su capacidad de absorción de agua de acuerdo con la norma ASTM D 570-90 (2010). Cinco muestras de cada MC fueron cortadas con las dimensiones $76.2 \times 25.4 \times 5 \mathrm{~mm}$ y se calculó su peso medio inicial $\left(\mathrm{M}_{1}\right.$, precisión de $\left.1 \mathrm{mg}\right)$. Las probetas fueron sumergidas en agua destilada a temperatura ambiente $\left(23 \pm 1{ }^{\circ} \mathrm{C}\right)$, en diferentes intervalos de tiempo. Para controlar la temperatura se utilizó un baño termostatado Reciprocal Shaking Bath modelo 250 (Thermo Electron Corporation, USA). Las probetas se retiraron del baño y, luego de removerles el exceso de agua superficial con papel adsorbente, se pesaron para obtener el peso final $\left(\mathrm{M}_{2}\right)$. Para calcular el porcentaje peso ganado, se usó la ecuación (1):

$\Delta M(\%)=\frac{M_{2}-M_{1}}{M_{1}} \times 100$

\section{Microscopia ESEM}

Las micrografías de la superficie fracturada de los $M C$ después de realizada la prueba de tensión fueron realizadas en un equipo Enviromental Scaning Electron Microscopy XL 30 ESEM (Philips, Eindoven, Holanda). La máquina tiene un detector de electrones secundarios gaseosos en una atmosfera de agua. Los resultados son mostrados a una magnificación de 500X.

\section{RESULTADOS Y DISCUSIÓN}

Las propiedades de un MC dependen de muchos factores como la resistencia y orientación del material de relleno en el compuesto, los tratamientos superficiales de las fibras, el procedimiento de fabricación del compuesto y el tipo de matriz, entre otros (Udaya Kiran, et al., 2007).

\section{Prueba de tensión}

El efecto de los tratamientos químicos en las propiedades mecánicas de los MC con las FSP, fique y vidrio, fue estudiado mediante la medida del módulo de Young, la resistencia última a la tracción y la elongación en 
el momento del rompimiento. Cuando se incrementa la rigidez de un material, aumenta su potencial para ser usado en aplicaciones que requieran estabilidad dimensional y capacidad de soportar altas cargas.

En la figura 1, se observa el comportamiento de la curva de tensión vs. la deformación de los diferentes MC. En la figura 1a, que corresponde a los MC con FSP tratadas y sin tratar (ST), se observa cómo el tratamiento con AA_EP muestra una mayor rigidez (superior módulo de Young), mientras que los otros dos tratamientos, además de aumentar la resistencia última a la tensión de los compuestos, incrementaron su deformación última o elongación al momento del rompimiento. En la figura 1b. se expone una comparación entre los resultados de la prueba mecánica de los MC con FSP, fibras de vidrio y fique. Es notoria la alta resistencia mostrada por los MC de fibras de vidrio (aprox. 400\% mayor que los de las fibras naturales), atribuida principalmente a su alta hidrofobicidad y consecuente mayor adhesión con la matriz polimérica. Sin embargo estos MC son frágiles pues se rompen inmediatamente después de alcanzar su resistencia máxima, tal como se observa en la figura 1b. Este mismo comportamiento se ha reportado en otros estudios (Mariana, et al., 2011). Las muestras elaboradas con fibras de fique presentaron un rompimiento irregular y alcanzaron más de dos veces la elongación que los MC producidos con FSP (Ver tabla 1).

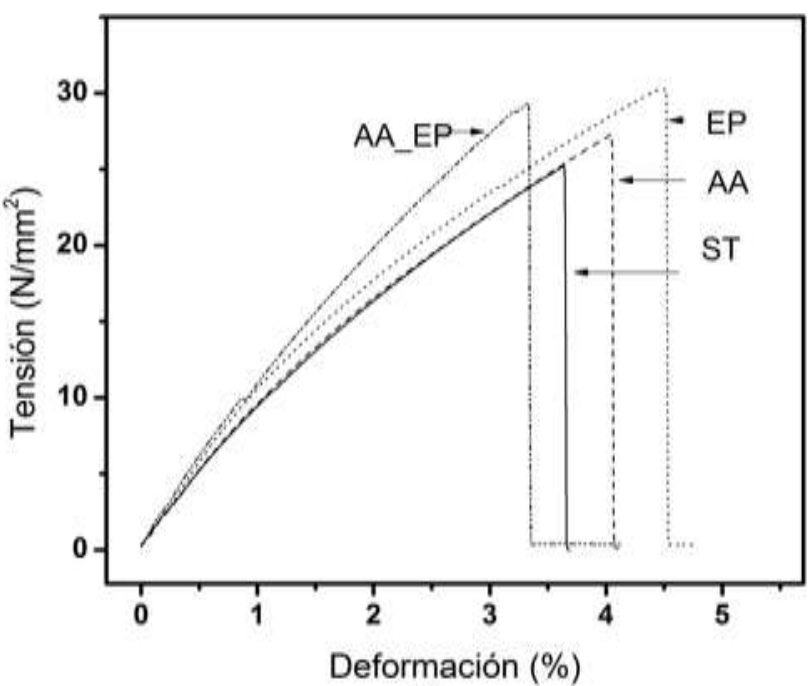

a)

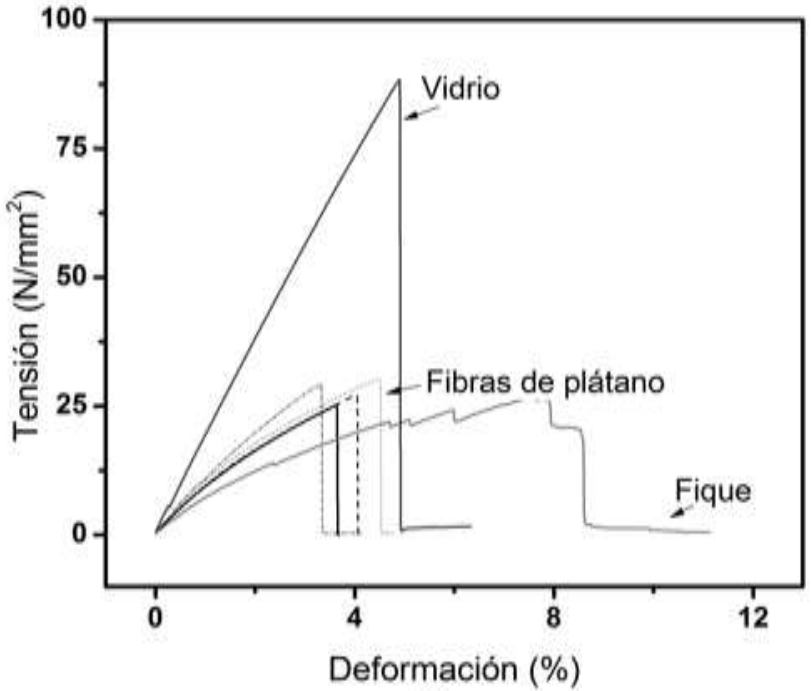

b)

Fig. 1: Resultados de la prueba de tensión. a) Materiales compuestos fabricados con las FSP b) Todos los materiales compuestos.

En la tabla 1 se comparan la resistencia última a la tracción, el módulo de Young y la elongación al momento del rompimiento de algunos de los $M C$ con fibras de plátano/banano fabricados en este estudio (FSP) y algunos de los datos reportados (Fibras de banano FB) recientemente para sistemas similares que usaron la técnica de moldeo manual. Como era de esperarse, las medidas para especímenes fabricados en poliéster sin relleno muestran los menores valores para estas propiedades.

Tabla 1: Comparación de las propiedades mecánicas de MC fabricados en matriz de poliéster, con FSP tratadas, sin tratar, fique y vidrio.

\begin{tabular}{|c|c|c|c|c|c|}
\hline Espécimen de $M C$ & $\begin{array}{l}\text { \% de fibra } \\
\text { en el MC }\end{array}$ & $\begin{array}{l}\text { Resistencia a la } \\
\text { tracción }(\mathrm{MPa})\end{array}$ & $\begin{array}{c}\text { Elongación } \\
(\%)\end{array}$ & $\begin{array}{l}\text { Módulo de } \\
\text { Young (GPa) }\end{array}$ & Referencia \\
\hline Poliéster sin relleno & -- & $\begin{array}{l}15,1 \\
31,5\end{array}$ & $\begin{array}{c}3,4 \\
--\end{array}$ & $\begin{array}{l}0,44 \\
0,63\end{array}$ & $\begin{array}{l}\text { (Murali Mohan Rao,et al.,2010) } \\
\text { (Ratna Prasad, et al., 2011) }\end{array}$ \\
\hline $\begin{array}{l}\text { FSP-Sin tratar, } \\
\text { tejida }\end{array}$ & & 27,7 & 3,6 & 1,04 & \\
\hline FSP-AA, tejida & 14,9 & 28,9 & 4,0 & 1,04 & Este trabajo \\
\hline FSP-EP, tejida & & 29,9 & 3,9 & 1,13 & \\
\hline FSP-AA_EP, tejida & & 30,7 & 3,8 & 1,23 & \\
\hline FB sin tratar & & 32.9 & -- & 0,68 & \\
\hline FB $0,3 \%$ silano & 20,0 & 35,5 & -- & 0,79 & (Pothan, et al., 1997) \\
\hline FB $1 \%$ silano & & 37,1 & -- & 0,52 & \\
\hline Fique, tejido & 14,9 & 18,3 & 7,5 & 0,95 & \\
\hline Vidrio, tela & 23,2 & 95,4 & 5,5 & 1,94 & Este trabajo \\
\hline
\end{tabular}

En cuanto a las FSP, se observa cómo el tratamiento con AA_EP aumentó considerablemente la resistencia media a la tracción, seguido de los tratamientos con EP y AA. Respecto a los especímenes de MC producidos con FSP sin tratar, el incremento en el módulo de Young para aquellos tratados con AA_EP fue 
de $18,27 \%$, mientras que los fabricados con fibras tratadas con AA aumentaron en $10,43 \%$ en su porcentaje de elongación. Los citados tratamientos promueven la remoción de ceras, hemicelulosas y pectinas de la superficie de la fibra, así como los posibles entrecruzamientos y reemplazos de los grupos OH. En conjunto, se afectan la morfología y la composición química de la fibra. Después de los tratamientos la superficie de las fibras es mas lisa, lo que incrementó su homogeneidad y la hidrofobicidad de su superficie. Estos cambios afectan positivamente la interacción inter-facial fibra-matriz y mejoran las propiedades de los MC tal como también fue observado en otros estudios (Pothan, et al., 2002) (Shah, et al., 2013). Efectos similares fueron observados, cuando se tratan FSP con silano a diferentes porcentajes.

Otro aspecto a resaltar es que usualmente las bajas proporciones de fibra en el MC, están asociadas a un pobre comportamiento mecánico (Pothan, et al., 1997). Sin embargo, y a pesar de que en este trabajo fue utilizado el modo de fabricación por moldeo manual, que no permite contenidos de fibra por encima del 1415\% (Dittenber, et al., 2012), se lograron mejoras sensibles en las propiedades mecánicas de los MC respecto de las que se observaron en los especímenes sin tratar. Debido a que el incremento en el porcentaje de fibra mejora la unión con la matriz y facilita la distribución de las cargas de tensión (Udaya Kiran, et al., 2007), sería de esperar que, usando otra técnica de fabricación que permita incrementar el porcentaje de fibra, los MC fabricados con FSP modificadas lograrían desempeños mecánicos superiores. De hecho, en la tabla 1 se puede observar que para las FB (carga del 20\%), hay un incremento en la resistencia última a la tracción y del módulo de Young.

\section{Prueba de impacto}

La Tabla 2 expone los resultados de las medidas de impacto (ensayo Izod) de los especímenes de MC que fueron fabricados en este trabajo y comparadas con un estudio que también utilizó matriz de poliéster y fibras de banano modificadas superficialmente con diferentes productos químicos. Para estas dos investigaciones las muestras con fibras naturales modificadas presentan una menor resistencia al impacto, lo cual se interpreta como un efecto de la disminución de la elasticidad debida a la mayor rigidez propiciada por los tratamientos químicos y la mayor adhesión fibra-matriz, que se alcanza mediante algunas alteraciones superficiales de las fibras. Esto a su vez disminuye la capacidad de deformación plástica de la matriz. Tal efecto puede ser también muestra del alto valor de impacto alcanzado por los MC de fibra de fique, a diferencia de la prueba de tensión. Los MC de fibra de vidrio, como era de esperarse, tienen propiedades de tenacidad superiores en comparación con las fibras naturales.

Tabla 2: Comparación de diferentes resultados de la prueba de tensión de especímenes de materiales compuestos.

\begin{tabular}{ccc}
\hline Tratamiento a la fibra & Impacto $\mathrm{kJ} / \mathrm{m}^{2}$ & Referencia \\
\hline Poliéster & 9,0 & (Idicula, et al., 2005) \\
\hline Sin tratamiento & 21,1 & \\
AA & 12,5 & Este trabajo \\
AA_EP & 16,5 & \\
EP & 12,5 & \\
\hline Sin tratamiento & 36,9 & \\
$0,5 \% \mathrm{NaOH}$ & 13,9 & (Pothan, et al., 2002) \\
$1 \% \mathrm{NaOH}$ & 6,1 & \\
Silano & 28,1 & \\
Acetilación & 23,9 & Este trabajo \\
\hline Fique & 48,7 & \\
Vidrio & 80,1 &
\end{tabular}

\section{Absorción de agua}

La reducción de la capacidad de absorción de agua de los MC aumenta su resistencia a la degradación cuando son expuestos a condiciones ambientales como humedad, luz solar, cambios de temperatura o microorganismos, preservando sus propiedades mecánicas y su estabilidad dimensional $(\mathrm{H}$. J. Kim, et al., 2006). La cantidad de absorción de agua de los compuestos depende del contenido de fibra, temperatura, orientación y tipo de fibras, permeabilidad, área expuesta de la superficie, porosidad e hidrofobicidad de cada uno de los componentes, entre otros factores (Sreekumar, et al., 2008). Para un mismo tipo de fibra, esta característica puede variar de acuerdo a su longitud y entramado dentro de la matriz, siendo mayor la absorción de humedad para rellenos con fibras largas o con fibras tejidas, arreglos que facilitan la difusión del agua dentro del MC (Sumaila, et al., 2013). La figura 2 muestra el porcentaje de ganancia de peso en función del tiempo (ver ecuación 1), después de sumergir en agua los diferentes MC a temperatura ambiente $\left(23^{\circ} \mathrm{C}\right)$. 
Los MC producidos con fibra de vidrio, el cual es un material altamente hidrofóbico, son los que presentan la menor absorción de agua (menor al 0,5\%). Entre los MC de fibras naturales, los de mejor comportamiento fueron los que usaron FSP tratadas, y, entre ellos, la menor absorción se observó en los hechos con FSP modificadas con AA_EP, lo que puede ser atribuido a una modificación efectiva mayor causada por el efecto sinérgico de estos dos químicos en la superficie de la fibra. En conjunto la reducción de la afinidad por el agua de las fibras naturales tratadas, hizo que sus MC a su vez disminuyeran entre dos y tres veces su capacidad de absorción al compararlos con los fabricados con las fibras sin tratar, hecho que hace prever que serían de mayor duración cuando sean expuestos a la intemperie. Por su parte, los compuestos con fibras de fique fueron los más afines al agua y, consecuentemente, de menor adhesión con la matriz y probablemente de menor resistencia a ambientes húmedos.

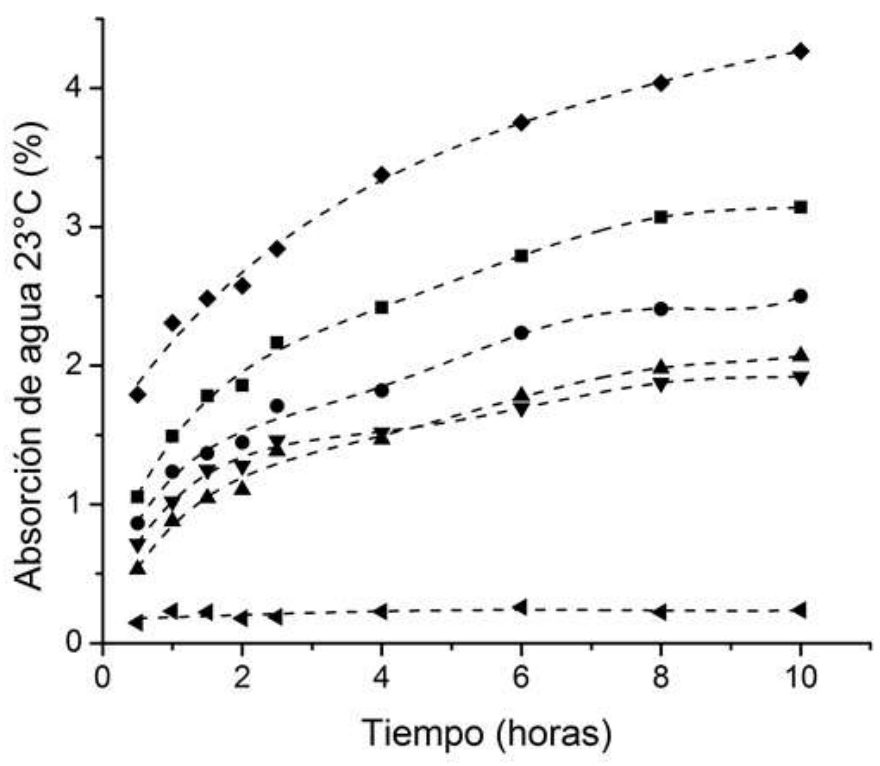

Fig. 2: Porcentaje de absorción de agua de los diferentes tipos de compuestos. a) Agua a temperatura ambiente $23^{\circ} \mathrm{C} \backsim \mathrm{ST} \bullet \mathrm{AA} \triangle \mathrm{EP} \nabla \mathrm{AA} E \mathrm{EP} \diamond \mathrm{F}\langle\mathrm{V}$

\section{Microscopia ESEM}

En la figura 3 se muestran las micrografías ESEM de la superficie fracturada de los MC, después de realizada la prueba de tensión, las cuales muestran visualmente parte de los análisis realizados anteriormente. La figura 3a. muestra la fractura de un MC de fique, en donde se puede ver que las fibras muestran un alto desplazamiento. Para el caso de los MC de fibras tratadas con epiclorhidrina, en la figura $3 \mathrm{~b}$ se observa que este desplazamiento es menor, mientras que en los fabricados con fibra de vidrio este efecto es pequeño (figura 3c). Tales deslizamientos o desplazamientos se pueden asociar a un diferente grado de adhesión de las fibras con la matriz poliestérica; se encontró que el menor deslizamiento se logra con las fibras de fique, mientras que con las de vidrio este fue el mayor entre todas las comparaciones.

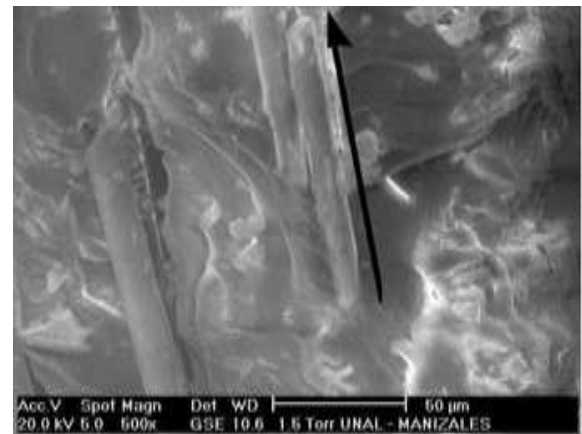

a)

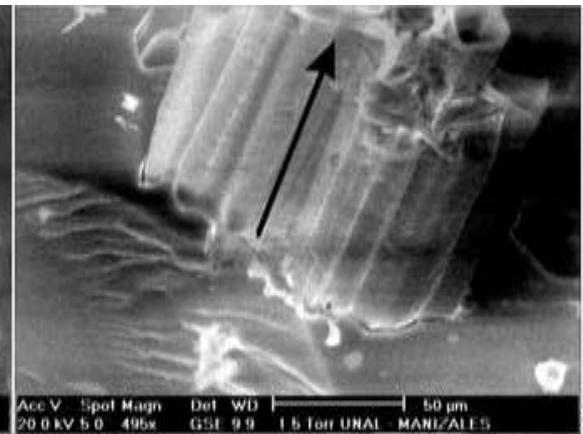

b)

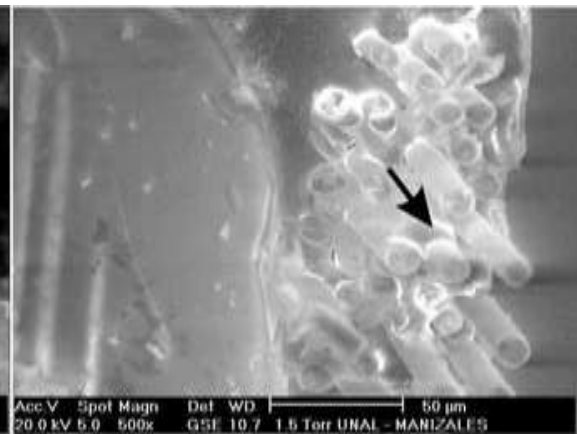

c)

Fig. 3: Micrografías ESEM de los MC a) fique b) FSP_EP y c) vidrio. 


\section{CONCLUSIONES}

Se fabricaron piezas de materiales compuestos, con FSP crudas y tratadas químicamente, fique y vidrio usando la técnica de moldeo manual. Su comportamiento fue comparado mediante pruebas de tensión, impacto, absorción de agua y micrografías ESEM. Los MC con FSP tratadas mostraron una reducción importante en la absorción de agua, lo que repercutió favorablemente en el aumento de las propiedades mecánicas de tensión, respecto de aquellos que no fueron tratados.

Entre los tratamientos realizados, el que usó la mezcla de anhídrido acético y epiclorhidrina (AA_EP) fue superior a los de cada químico por separado pues sus MC tuvieron un desempeño mejor en las pruebas de tensión y absorción de agua. Esto se explica por el efecto combinado de sustitución de los grupos $\mathrm{OH}$ de las fibras por efectos de la acetilación y entrecruzamiento por el anhídrido acético y la epiclorhidrina respectivamente.

Todos los MC fabricados con fibras tratadas fueron menos tenaces en la prueba de impacto. Aparentemente, la ganancia de adhesión fibra-matriz trae como consecuencia menor elasticidad de los MC, tal como se ha reportado en otros trabajos publicados para sistemas de FSP-matriz poliestérica (Tabla 2).

Aunque los compuestos elaborados con fibras de vidrio muestran propiedades superiores, el aumento de propiedades como la rigidez y la hidrofobicidad obtenido por las FSP tratadas, extiende su viabilidad para aplicaciones en empaques, donde la hidrofobicidad, bajo peso, buena tensión y biodegrabilidad son características importantes. Además los MC producidos con FSP sin tratar, mostraron mejores propiedades que los de fibra de fique. Dado que dado que la fibra de fique es uno de los materiales más utilizados y reconocidos en el mercado internacional, se evidencia el potencial de las FSP para diversos usos.

\section{AGRADECIMIENTOS}

Los autores agradecen el apoyo financiero de COLCIENCIAS (programa "Jóvenes investigadores e innovadores, convocatoria 566/2012) y a la Universidad Nacional de Colombia, Sede Manizales, proyecto HERMES Código: 18617: Gestión Tecnológica e Innovación en Biotecnología Industrial.

\section{REFERENCIAS}

Dittenber, D. B. y GangaRao, H. V. S., Critical review of recent publications on use of natural composites in infrastructure, Composites Part A: Applied Science and Manufacturing, 43 (8), 1419-1429, (2012).

FAO, Año internacional de las fibras naturales, http://www.naturalfibres2009.org/es/, Octubre (2012).

Flieger, M. y otros cuatro autores, Biodegradable plastics from renewable sources, Folia Microbiologica, 48 (1), 27-44, (2003).

Freier, T. y otros tres autores, Controlling cell adhesion and degradation of chitosan films by $\mathrm{N}$-acetylation, Biomaterials, 26 (29), 5872-5878, (2005).

Gutiérrez, I. y otros tres autores, Influencia del Tratamiento con Vapor Sobre la Estructura $y$ Comportamiento Físico-Mecánico de Fibras de Plátano, Información tecnológica, 16, 15-21, (2005).

Idicula, M. y otros cuatro autores, A study of the mechanical properties of randomly oriented short banana and sisal hybrid fiber reinforced polyester composites, Journal of Applied Polymer Science, 96 (5), 16991709, (2005).

Johansson, C. y otros diez autores, Renewable Fibers And Bio-based Materials for Packaging Applications, BioResources, 7 (2), 1-47, (2012).

Kim, D.-Y., Nishiyama, Y. y Kuga, S., Surface acetylation of bacterial cellulose, Cellulose, 9 (3-4), 361-367, (2002).

Kim, H. J. y Seo, D. W., Effect of water absorption fatigue on mechanical properties of sisal textile-reinforced composites, International Journal of Fatigue, 28 (10), 1307-1314, (2006).

Kuniak, L. y Marchessault, R. H., Study of the Crosslinking Reaction between Epichlorohydrin and Starch, Starch - Stärke, 24 (4), 110-116, (1972). 
Mariana, E. y otros tres autores, Influencia del Recubrimiento de las Fibras de Vidrio sobre la Efectividad de la Reacción de Copolimerización Propileno-Vidrio, Información tecnológica, 22, 77-82, (2011).

Mohanty, A. K., Drzal, L. T. y Misra, M. Natural Fibers, Biopolymers, and Biocomposites, (2005).

Murali-Mohan-Rao, K., Mohana-Rao, K. y Ratna-Prasad, A. V., Fabrication and testing of natural fibre composites: Vakka, sisal, bamboo and banana, Materials \& Design, 31 (1), 508-513, (2010).

Oliveira, F.y otros tres autores, Surface Modification of Banana Fibers by DBD Plasma Treatment, Plasma Chemistry and Plasma Processing, 32, 259-273, (2012).

Pothan, L. A., George, J. y Thomas, S., Effect of fiber surface treatments on the fiber-matrix interaction in banana fiber reinforced polyester composites, Composite Interfaces, 9 (4), 335-353, (2002).

Pothan, L. A., Thomas, S. y Neelakantan, N. R., Short Banana Fiber Reinforced Polyester Composites: Mechanical, Failure and Aging Characteristics, Journal of Reinforced Plastics and Composites, 16 (8), 744765, (1997).

Ratna-Prasad, A. V. y Mohana-Rao, K., Mechanical properties of natural fibre reinforced polyester composites: Jowar, sisal and bamboo, Materials \& Design, 32 (8-9), 4658-4663, (2011).

Rodríguez, L., Suárez, O. y Pareja, A. Cadena de valor en el sector primario: El caso del plátano Universidad Nacional de Colombia: Sede Manizales, (2011).

Shah, H., Srinivasulu, B. y Shit, S. C., Influence of banana fibre chemical modification on the mechanical and morphological properties of woven banana fabric/unsaturated polyester resin composites, Polymers from Renewable Resources, 4 (2), 61-65, (2013).

Sreekumar, P. A. y otros cuatro autores, Mechanical and water sorption studies of ecofriendly banana fiberreinforced polyester composites fabricated by RTM, Journal of Applied Polymer Science, 109 (3), 15471555, (2008).

Sumaila, M., Amber, I. y Bawa, M., Effect of fiber length on the physical and mechanical properties of random oreinted, nonwoven short banana (musa balbisiana) fibre/epoxy composite, Asian Journal of Natural and Applied Sciences, 2 (1), 39-49, (2013).

Udaya-Kiran, C. y otros tres autores, Tensile Properties of Sun Hemp, Banana and Sisal Fiber Reinforced Polyester Composites, Journal of Reinforced Plastics and Composites, 26 (10), 1043-1050, (2007).

Zeng, X. y Ruckenstein, E., Control of Pore Sizes in Macroporous Chitosan and Chitin Membranes, Industrial \& Engineering Chemistry Research, 35 (11), 4169-4175, (1996). 